\title{
Pathological Complete Remission in Young Colon Cancer Patient with a Large Liver Metastasis after FOLFOX-4/Bevacizumab Treatment-A Case Report
}

\author{
Elīna Skuja ${ }^{1}$, Arnis Āboliṇš ${ }^{1,2}$, Ilze Priedīte ${ }^{1}$, Gunta Purkalne ${ }^{1}$, Ilze Štrumfa ${ }^{2}$, Jānis Vilmanis ${ }^{1}$, \\ Dagnija Kalniete $^{1,2}$, Edvīns Miklaševičs ${ }^{1,2}$, Jānis Gardovskis ${ }^{1,2}$ \\ ${ }^{1}$ Pauls Stradins Clinical University Hospital, Riga, Latvia; ${ }^{2}$ Hereditary Cancer Institute, Riga Stradins University, Riga, Latvia. \\ Email: elina.skuja@stradini.lv
}

Received March $4^{\text {th }}, 2012$; revised April 16 ${ }^{\text {th }}, 2012$; accepted May 24 $4^{\text {th }}, 2012$

\begin{abstract}
Introduction: Pathological complete remission of liver metastases is a rare colon cancer treatment outcome with increased 5-year survival of 76\%. Case Report: Metastatic colorectal cancer patient with pathological complete remission of large hepatic metastasis after palliative chemotherapy in combination with bevacizumab is presented. Solitary liver metastasis measuring $8 \mathrm{~cm}$ was observed in computed tomography (CT) scan before combined treatment. The best radiological response during treatment with FOLFOX-4 and bevacizumab therapy was partial remission and patient underwent partial hepatectomy. Since the operation material was free of viable adenocarcinoma cells the effect of FOLFOX-4 in combination with bevacizumab treatment was interpreted as the pathological complete remission. Conclusion: Use of combination chemotherapy and targeted therapy with the aim to reduce initially unresectable liver metastasis is the best option to achieve complete pathological remission and significantly prolong survival.
\end{abstract}

Keywords: Metastatic Colorectal Cancer; Pathological Complete Remission; Bevacizumab

\section{Introduction}

There are about 410,000 new cases of colorectal cancer in Europe every year. Most of patients with colorectal cancer (CRC) are over 65 years of age and the disease is rare before the age of 45 (2 per 100,000/year). Approximately $25 \%$ present with metastases at initial diagnosis and almost $50 \%$ of patients with CRC will develop metastases, contributing to the high mortality rates reported for metastatic colorectal cancer (mCRC) [1]. Despite recent advances in the medical treatment of mCRC, which include oxaliplatin- and irinotecan-based first-line regimens and the increasing use of targeted monoclonal antibodies, survival rates for patients with mCRC remain unacceptably low [2]. The standard of care is palliative chemotherapy in order to prolong overall survival and control symptoms.

About $13 \%$ of patients with initially unresectable metastases can become resectable after multidrug chemotherapy [3], that can improve progression free and overall survival: 5 -year survival rates are in the range of $21 \%$ to $58 \%$ in patients with colorectal cancer liver metastases after surgical resection [4].

Among all patients who underwent liver metastasis resection, $4 \%$ present a rare clinically significant situation-pathological complete remission (pCR), a complete absence of residual cancer cells on examination by a pathologist [5]. More recently, the addition of monoclonal antibodies (bevacizumab or cetuximab) to cytotoxic chemotherapy has increased the rate of tumor response, suggesting that the addition of these agents could improve resectability rate $[5,6]$. Patients who achieve pathological complete remission, which is most often defined as the complete disappearance of cancer cells in the tumor area after preoperative therapy, have a better prognosis than those who do not [6]. In recent years, several studies have been performed to find markers to predict the probability of patients achieving pathologic complete remission, including routine clinical and pathological characteristics, biomarkers, gene expression profiling and imaging techniques [6,7]. Because of there has been relatively small account of prospective randomized trials, the gold standard to predict a response to preoperative therapy is still missing.

The objective of the research is to analyze the results of a clinical case and to identify possible predictive and prognostic factors which might affect treatment outcome. 


\section{Case Report}

Thirty five-year-old man was urgently hospitalized at surgical department of District Hospital with clinically diagnosed bowel obstruction and has undergone Hartmann's type resection of distal descendent colon (sigma). The patient reported a 1-year history of constipation and abdominal pain but did not receive any treatment before presentation. Whereas during the operation was found malignancy it was decided to continue diagnostic procedures and treatment in University hospital with experience in colorectal cancer management.

Patient was hospitalized at Pauls Stradins Clinical University Hospital Oncology Clinic. Abdominal computed tomography (CT) scan showed a large unresectable solitary liver metastasis in right lobe (segment 8) with 8 $\mathrm{cm}$ as the largest diameter (Figure 1). A chest CT scan showed no evidence of lung metastases, and the cardiac, mediastinal, pleural, and bony structures were normal. Carcinoembryonic antigen (CEA) level was $4.2 \mathrm{ng} / \mathrm{ml}$-a normal reference is $0.0-5.0 \mathrm{ng} / \mathrm{ml}$. Results of a complete blood count, plasma levels of electrolytes, tests of coagulation and kidney and liver function, and a urinalysis were normal. The patient's family has no cancer history.

Poorly differentiated adenocarcinoma that invades through the muscularis propria into the subserosa and metastases in 7 regional lymph nodes were found in operation material corresponding to pT3N2bG3R0. Immunohistochemical staining was positive for CK20 and CDX2 and negative for TP53 staining. Proliferation fraction by Ki-67 protein expression was $73 \%$. Immunohis-

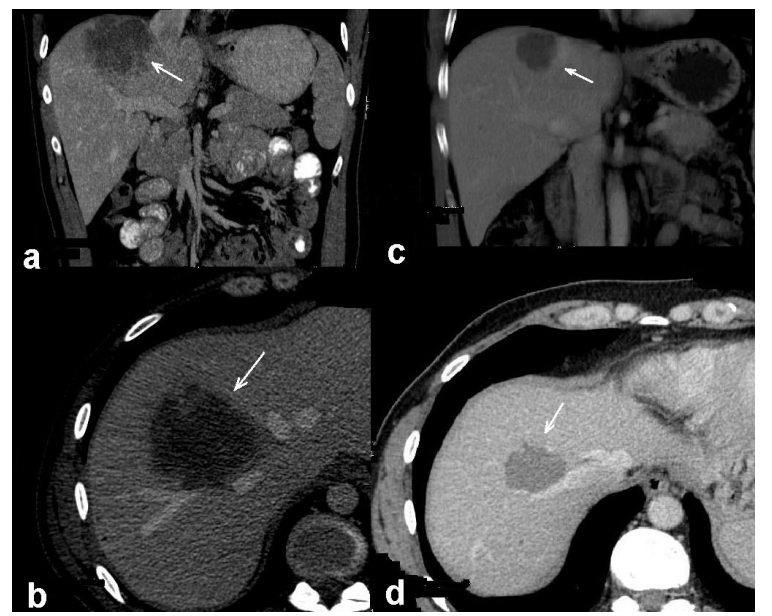

Figure 1. CT scans before (a), (b) and after (c), (d) neoadjuvant treatment. Solitary large initially unresectable liver metastasis marked with arrow heads in right lobe is seen (a), (b). After FOLFOX plus bevacizumab treatment the liver metastasis was reduced in size by $50 \%$. Radiological partial response of metastasis marked with arrow heads can be seen on CT scan (c), (d). The operation material revealed pathological complete remission. tochemical analysis of two mismatch-repair genes' (MSH2 and MSH6) products showed isolated loss of nuclear MSH6 immunoreactivity, suggesting loss-offunction mutation in the MSH6 gene. As the isolated loss of nuclear immunoreactivity for MSH6 in the tumor indicates for a putative germline mutation [8], the MSH6 gene was sequenced but no clinically significant germline mutation was found. Tumor tissue was checked for predominant somatic mutation (KRAS 12th, 13th and 61st codons, BRAF V600E, TP53 5th - 8th exons, PIK3CA 9th and 10th exons and PTEN whole coding region) and only one mutation G61H in the KRAS gene was found.

It was decided to initiate neoadjuvant chemotherapy with FOLFOX4/bevacizumab to make initially unresectable hepatic colon cancer metastasis resectable. The patient received eight infusions of neoadjuvant chemotherapy with FOLFOX-4 and four infusions of bevacizumab every 2 weeks at 4-month period. The mild sensory peripheral neuropathy, grade 1 neuthropenia and grade 1 hepatotoxicity was observed during treatment period.

Control CT scan showed partial remission-the tumor was decreased by $50 \%$ with $4 \mathrm{~cm}$ in greatest dimension (Figure 1). Neoadjuvant chemotherapy plus biological agent turned initially unresectable metastasis resectable, and patient became eligible for potentially curative surgery. The patient underwent a partial hepatectomy (resection of segment 8). Intraoperative ultrasonography (IOUS) during surgery excluded additional liver metastases. The cut-surface of the resected specimen from S8 showed a homogenous yellow-white mass. The size of solitary lesion, as described in the gross pathology report, was $3.4 \mathrm{~cm}$ by $2.3 \mathrm{~cm}$. Microscopically, most of the specimen were replaced with necrotic tissue and granulomatous inflammation. Shadows of cytokeratin 20positive and CDX2-positive cells were found in the necrosis. The operation material was free of viable adenocarcinoma cells. Since no viable tumor cells were observed in any of the whole sections of the resected specimen, entirely submitted for microscopic evaluation, the effect of FOLFOX-4 in combination with bevacizumab treatment on the metastatic colon cancer was interpreted as the pathological complete remission.

The patient recovered from surgery without any complications, completed additional four infusions of postoperative FOLFOX-4 chemotherapy. At this moment, 14 months since hepatic surgery, the examinations showed no evidence of disease.

\section{Discussion}

This case report shows a complete pathological remission in primary metastatic colorectal cancer patient with un- 
favorable prognosis and a small chance to recovery. In a recent study the probability of complete pathological response is described based on four predictive factors in patients treated with preoperative chemotherapy-age < 60 years, size $<3 \mathrm{~cm}$, preoperative CEA $<30 \mathrm{ng} / \mathrm{ml}$, objective radiological response [6]. According to these data the patient had $10 \%$ probability of pCR of liver metastasis. In this study, the pCR of liver metastases in patients after resection was associated with a 5-year overall survival rate of $76 \%$ compared to $45 \%$ in patients without a pCR.

There are published many clinical factors to predict outcome after resection of colorectal liver metastasesnodal status, disease-free interval from the primary to discovery of the liver metastases, number of metastases, preoperative CEA level, size of the metastases. Regarding to the clinical risk score the patient's prognosis is calculated with 5-year survival rate of $20 \%$ [9]. In spite of unfavorable clinical factors the complete pathological remission of liver metastasis was achieved which significantly improves the patient's prognosis.

In our case the mutations shown in cancer cells aren't clinically significant. None of driver mutations (KRAS, BRAF, PTEN, TP53 and PIK3CA) was found. It is tempting to speculate that the good treatment results are due to lack of any clinically relevant mutation combined with the young age, single liver lesion, normal CEA level and loss of MSH6 staining in cancer tissue. DNA mismatch repair dysfunction could change response to treatment DNA damaging agents. Several studies have shown that defects in mismatch repair genes result in increased cisplatin but not oxaliplatin resistance [10].

Due to wild-type BRAF and KRAS in cancer tissue, initially it was considered to use an anti-EGFR monoclonal antibody cetuximab according to guidelines [1]. The first reason why it was decided in favor of antiVEGF antibody bevacizumab was the fact that it in combination with oxaliplatin-based regimen chemotherapy increase the rates of radical liver surgery $[5,6]$, and the second one-was controversial role of the G61H mutation in the KRAS gene on resistance to cetuximab [11]. Costs of medication were taken into account. It should be noted that standard palliative chemotherapy in Latvia is oxaliplatin or irinotecan containing regiments, but monoclonal antibodies are not reimbursed.

The prediction of a complete pathological response of metastases after multidrug chemotherapy is important, but it is quite difficult, since there is no clear correlation between a pathological response and a radiological response. That patient contrast enhanced CT scan revealed partial radiological response with tumor shrinkage by $50 \%$ after chemotherapy. It must be noted that total necrosis of tumor cells does not imply disappearance of metastasis in pre-operative imaging and does not necessarily correspond to radiological complete remission $[6,7]$. By contrast, a complete radiological response is not always associated with a complete pathologic response [12].

\section{Conclusion}

Metastatic colorectal cancer is potentially fatal disease with short survival. Use of combination chemotherapy plus targeted therapy with the aim to reduce initially unresectable liver metastasis is the only option to achieve complete pathological remission and significantly prolong survival.

\section{Acknowledgements}

Part of this work was supported by The National Research Programme "Development of new prevention, treatment, diagnostics means and practices and biomedicine technologies for improvement of public health".

\section{REFERENCES}

[1] E. Van Cutsem, J. Oliveira and on Behalf of the ESMO Guidelines Working Group, “Advanced Colorectal Cancer: ESMO Clinical Recommendations for Diagnosis, Treatment and Follow-Up,” Annals of Oncology, Vol. 20, No. 4, 2009, pp. iv61-iv63. doi:10.1093/annonc/mdp130

[2] L. B. Saltz, S. Clarke, E. Díaz-Rubio, W. Scheithauer, A. Figer, R. Wong, S. Koski, M. Lichinitser, T. S. Yang, F. Rivera, F. Couture, F. Sirzén and J. Cassidy, "Bevacizumab in Combination with Oxaliplatin-Based Chemotherapy as First-Line Therapy in Metastatic Colorectal Cancer: A Randomized Phase III Study," Journal of Clinical Oncology, Vol. 26, No. 12, 2008, pp. 2013-2019. doi:10.1200/JCO.2007.14.9930

[3] R. Adam, E. Avisar, A. Ariche, S. Giachetti, D. Azoulay, D. Castaing, F. Kunstlinger, F. Levi and F. Bismuth, "Five-Year Survival Following Hepatic Resection after Neoadjuvant Therapy for Unresectable Colorectal Liver Metastases,” Annals of Surgical Oncology, Vol. 8, No. 4, 2001, pp. 347-353. doi:10.1007/s10434-001-0347-3

[4] M. Hayashi, Y. Inoue, K. Komeda, T. Shimizu, M. Asakuma, F. Hirokawa, Y. Miyamoto, J. Okuda, A. Takeshita, Y. Shibayama and N. Tanigawa, "Clinicopathological Analysis of Recurrence Patterns and Prognostic Factors for Survival after Hepatectomy for Colorectal Liver Metastasis,” BMC Surgery, Vol. 10, 2010, p. 27. doi:10.1186/1471-2482-10-27

[5] D. G. Blazer III, Y. Kishi, D. M. Maru, S. Kopetz, Y. S. Chun, M. J. Overman, D. Fogelman, C. Eng, D. Z. Chang, H. Wang, D. Zorzi, D. Ribero, L. M. Ellis, K. Y. Glover, R. A. Wolff, S. A. Curley, E. K. Abdalla and J. N. Vauthey, "Pathologic Response to Preoperative Chemotherapy: A New Outcome End Point after Resection of Hepatic Colorectal Metastases,” Journal of Clinical Oncology, 
Vol. 25, No. 33, 2008, pp. 5344-5351.

doi:10.1200/JCO.2008.17.5299

[6] R. Adam, D. A. Wicherts, R. J. de Haas, T. Aloia, F. Lévi, B. Paule, C. Guettier, F. Kunstlinger, V. Delvart, D. Azoulay and D. Castaing, "Complete Pathologic Response after Preoperative Chemotherapy for Colorectal Liver Metastases: Myth or Reality?” Journal of Clinical Oncology, Vol. 26, No. 10, 2008, pp. 1635-1641. doi:10.1200/JCO.2007.13.7471

[7] G. Y. Locker, S. Hamilton, J. Harris, J. M. Jessup, N. Kemeny, J. S. Macdonald, M. R. Somerfield, D. F. Hayes and R. C. Bast, "ASCO 2006 Update of Recommendations for the Use of Tumor Markers in Gastrointestinal Cancer,” Journal of Clinical Oncology, Vol. 24, No. 33, 2006, pp. 5313-5327. doi:10.1200/JCO.2006.08.2644

[8] M. J. Berends, Y. Wu, R. H. Sijmons, R. G. Mensink, T. van der Sluis, J. M. Hordijk-Hos, E. G. de Vries, H. Hollema, A. Karrenbeld, C. H. Buys, A. G. van der Zee, R. M. Hofstra and J. H. Kleibeuker, "Molecular and Clinical Characteristics of MSH6 Variants: An Analysis of 25 Index Carriers of a Germline Variant," American Journal of Human Genetics, Vol. 70, No. 1, 2002, pp. 26-37. doi:10.1086/337944

[9] Y. Fong, J. Fortner, R. L. Sun, M. F. Brennan and L. H. Blumgart, "Clinical Score for Predicting Recurrence after Hepatic Resection for Metastatic Colorectal Cancer:
Analysis of 1001 Consecutive Cases,” Annals of Surgery, Vol. 230, No. 3, 1999, pp. 309-321. doi:10.1097/00000658-199909000-00004

[10] A. Vaisman, M. Varchenko, A. Umar, T. A. Kunkel, J. I. Risinger, J. C. Barrett, T. C. Hamilton and S. G. Chaney. "The Role of hMLH1, hMSH3, and hMSH6 Defects in Cisplatin and Oxaliplatin Resistance: Correlation with Replicative Bypass of Platinum-DNA Adducts,” Cancer Research, Vol. 58, No. 16, 1998, pp. 3579-3585.

[11] W. De Roock, D. J. Jonker, F. Di Nicolantonio, A. Sartore-Bianchi, D. Tu, S. Siena, S. Lamba, S. Arena, M. Frattini, H. Piessevaux, E. Cutsem, C. J. O’Callaghan, S. Khambata-Ford, J. R. Zalcberg, J. Simes, C. S. Karapetis, A. Bardelli and S. Tejpar, "Association of KRAS p.G13D Mutation with Outcome in Patients with ChemotherapyRefractory Metastatic Colorectal Cancer Treated with Cetuximab," JAMA, Vol. 304, No. 16, 2010, pp. 1812 1820. doi:10.1001/jama.2010.1535

[12] R. C. Auer, R. R. White, N. E. Kemeny, L. H. Schwartz, J. Shia, L. H. Blumgart, R. P. Dematteo, Y. Fong, W. R. Jarnagin and M. I. D'Angelica, "Predictors of a True Complete Response among Disappearing Liver Metastases from Colorectal Cancer after Chemotherapy," Cancer, Vol. 116, No. 6, 2010, pp. 1502-1509. doi:10.1002/cncr.24912 